\title{
Synthèse des méthodes actuelles de détermination de la contamination atmosphérique par les transuraniens*
}

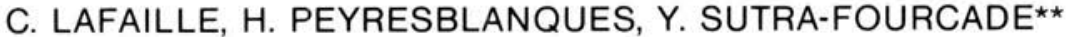

\author{
RÉSUMÉ
}

\begin{abstract}
Le contrôle de l'activité atmosphérique doit prendre en compte différentes données qui concernent notamment la configuration géographique, les caractéristiques de ventilation, l'influence de la radioactivité naturelle et les types de matériels utilisés.
\end{abstract}

Les spécialistes en la matière se penchent sur ces problèmes depuis pratiquement la naissance de l'industrie nucléaire pour aboutir à des mesures aussi représentatives que possible.

Le présent document a pour objet de faire le point de l'état d'avancement de ces études et d'énumérer les méthodes qui paraissent devoir être retenues.

\section{ABSTRACT}

The monitoring of atmospheric activity must consider various data especially on the geographical conditions, the ventilation characteristics, the effect of natural radioactivity and the types of equipment used. These problems have been studied practically since the advent of nuclear industry in order to reach measurements as representative as possible.

The progress of these studies and the methods that should be retained are reviewed.

\section{INTRODUCTION}

Parmi les mesures physiques devant conduire à la détermination des risques radiologiques, l'évaluation de l'activité atmosphérique pose certainement les problèmes les plus complexes. Ceci provient essentiellement de la mauvaise connaissance de la cinématique des aérosols et de l'interférence de ceux-ci avec des éléments naturels qui viennent perturber la représentativité des estimations effectuées. On comprendra ainsi l'acharnement mis par les spécialistes en la matière pour mettre au point des dispositifs capables de prendre en compte ces différents éléments. Malgré la réalisation d'appareillages souvent ingénieux, les résultats obtenus n'ont pas toujours donné les satisfactions espérées.

* Communication présentée aux journées d'études "Plutonium et radioprotection", organisées par la SFRP, Saclay, 14-16 juin 1983.

** COGEMA, Service de protection contre les rayonnements, Etablissement de Marcoule, BP 170, 30205 Bagnols-sur-Ceze Cedex. 
Actuellement, après des années d'études plus ou moins fructueuses, un certain nombre de solutions peuvent, néanmoins, être retenues et il convient de les recenser en fonction des divers paramètres qui interviennent dans les différents aspects de cette technologie.

\section{BUT DU CONTROLE}

Le contrôle d'ambiance imposé par le décret 75-306 du 28 avril 1975 a pour but principal de protéger le personnel en donnant une alarme en cas de contamination atmosphérique décelable dans le local. Ce système d'alarme ne consiste pas seulement en un appareillage spécifique, mais doit également prendre en compte, dans une certaine mesure :

- la configuration du local ;

- l'implantation du matériel d'exploitation et, en particulier, les points sensibles du circuit, c'est-à-dire ceux susceptibles d'être le plus à l'origine d'une contamination ;

- l'organisation de l'exploitation (changement systématique des gants de boîte, cheminement du personnel et du matériel,...) ;

- la ventilation des locaux et l'implantation des bouches d'extraction et de soufflage ;

- l'utilisation de prélèvements d'air avec analyse différée soit à poste fixe, soit avec des appareils individuels ;

- la minutie et la fréquence des contrôles de contamination de surface ;

- la présence plus ou moins importante de radon et de thoron liée à la nature et à l'efficacité des filtres d'entrée, aux entrées parasites d'air extérieur et à la nature des matériaux de construction du local.

Le système d'alarme forme donc un tout: l'appareillage proprement dit et les éléments énumérés ci-dessus. L'ensemble doit donc être étudié globalement dès l'origine du projet. Néanmoins, il est évident que cet appareillage constitue le pivot du dispositif d'alarme et doit donc être surtout sensible et fiable. Cet appareillage comporte :

- une tête de prélèvement (de captation) ;

- une tuyauterie de liaison avec l'ensemble détecteur ;

- un dispositif de collection des poussières ;

- un ou plusieurs détecteurs de radioactivité ;

- une électronique avec, outre la génération des alarmes, les contrôles nécessaires au bon fonctionnement de l'ensemble ;

- un dispositif d'aspiration avec, éventuellement, une régulation de débit.

\section{MODALITES DU CONTROLE}

Comment effectuer un contrôle de contamination atmosphérique ? En amenant jusqu'au détecteur un échantillon représentatif. Malheureusement, suivant l'isotope considéré $\left({ }^{238} \mathrm{Pu}\right.$ par exemple) ou la composition isotopique du plutonium (forte proportion de ${ }^{240} \mathrm{Pu}$ par exemple), quelques particules par mètre cube suffisent pour atteindre la CMA. II est donc difficile de parler de répartition homogène dans l'atmosphère. Le dispositif de prélèvement ne peut présenter qu'une probabilité plus ou moins grande de collection des particules. En conséquence, il convient d'accroître cette probabilité en essayant de s'affranchir de l'inconvénient "radon". Pour cela : 
- le nombre et l'emplacement des points de prélèvement doivent être judicieusement choisis ;

- le débit de prélèvement doit être important pour accroître la probabilité de captation des particules ;

- le prélèvement doit être parfaitement "représentatif" au point de vue granulométrie (mais est-ce possible?) ;

- les poussières prélevées doivent parvenir, sans pertes, jusqu'au dispositif de collection ;

- le rendement de détection doit être aussi élevé que possible ;

- lappareillage doit permettre la discrimination des éléments de filiation du radon et du thoron.

\section{PROCEDES ET MOYENS DE CONTROLE}

\section{Détermination des points de prélèvement}

Des études, effectuées au CEA, Département de protection technique (DPT), et cautionnées par un groupe de travail, le GTR 4, permettront, grâce à des émissions d'aérosols, en continu ou par bouffées, de déterminer les temps de transfert des particules et leur concentration relative dans un local donné. Grâce à un appareillage susceptible d'être utilisé dans les Services de protection contre les rayonnements (SPR), ceux-ci seront alors à même d'avoir une vue plus exacte de ces transferts, donc de mieux implanter les têtes de prélèvements et d'en optimiser le nombre.

\section{Importance du débit d'aspiration}

Quelques chiffres pour donner une idée de la densité de particules nécessaires pour avoir $5 \mathrm{CMA}$.h en $10 \mathrm{~min}(30 \mathrm{CMA})^{1}$ :

Pu à $8 \%$ de $^{240} \mathrm{Pu}: 69$ particules de $1 \mu \mathrm{m}$ par $\mathrm{m}^{3}$ ou 0,51 particule de $5 \mu \mathrm{m} \mathrm{par} \mathrm{m}^{3}$. Pu à $20 \%$ de ${ }^{240} \mathrm{Pu}: 51$ particules de $1 \mu \mathrm{m}$ par $\mathrm{m}^{3}$ ou 0,42 particule de $5 \mu \mathrm{m}$ par $\mathrm{m}^{3}$. ${ }^{238} \mathrm{Pu}: 0,36$ particule de $1 \mu \mathrm{m}$ par $\mathrm{m}^{3}$ ou 0,0029 particule de $5 \mu \mathrm{m}$ par $\mathrm{m}^{3}$. $\mathrm{PuO}_{2}$ à $5 \%$ de $^{240} \mathrm{Pu}: 220$ particules de $1 \mu \mathrm{m}$ par $\mathrm{m}^{3}$ ou 1,8 particule de $5 \mu \mathrm{m}$ par $\mathrm{m}^{3}$.

Des valeurs aussi faibles montrent qu'il est nécessaire d'augmenter autant que possible le débit de prélèvement des appareils de mesure. La balise à grande sensibilité BFSAB doit, d'ailleurs, avoir un débit voisin de $1 \mathrm{~m}^{3}$ par minute.

\section{Représentativité granulométrique}

En ce qui concerne la représentativité de la tête de prélèvement, une étude est actuellement en cours au DPT sur un dispositif utilisable avec cette même balise. Une adaptation pourrait être faite pour d'autres appareils de débit différent. Rappelons qu'une étude réalisée par la société Bertin avait abouti à un ensemble performant mais d'un prix de revient élevé.

\section{Dépôt dans les canalisations}

Le dépôt des particules dans les conduits a déjà fait l'objet de nombreuses études, en particulier le rapport de J. CHARUAU qui contient de

1. II s'agit des CMA travailleurs selon le décret 66-450 du 20 juin 1966. 
nombreuses références [1]. II sera indispensable de calculer le diamètre des conduits pour chaque prélèvement, en fonction de la longueur, du débit et de la granulométrie des particules, ceci, afin de minimiser les pertes. Bien sûr, le plus simple est peut-être de supprimer les tuyauteries et de placer la tête de détection dans le local, mais on risque alors d'être amené à multiplier le nombre des appareils sans que l'accroissement de la sécurité soit toujours évident.

\section{Collection des poussières}

La collection des poussières s'effectue, dans la grande majorité des appareils, sur filtre fibreux ou sur membrane filtrante. Deux exceptions, cependant : le MD Pu qui collectait sur film scintillant adhésif et un appareil anglais assez ancien qui collectait sur ruban adhésif de bureau.

\section{Sensibilité}

L'amélioration de la sensibilité passe par l'augmentation du rendement de détection et surtout par la discrimination entre les éléments artificiels et naturels. Pour cela, différents principes sont utilisés :

\section{PRINCIPES DE DISCRIMINATION}

Pseudo coincidence $\alpha-\beta$. Certains descendants du radon et du thoron étant émetteurs $\alpha$ et $\beta$, une pseudo coïncidence permet de les distinguer des émetteurs $\alpha$ purs, donc de les éliminer (BFSAB, EAR 901, appareils Frieseke Hoepfner ou Berthold).

Spectrométrie $\alpha$ à pression atmosphérique. Grâce à une spectrométrie sur diode à barrière de surface, il est possible de différencier les émissions $\alpha$ dues à la filiation du radon ou thoron de celles dues au plutonium par exemple. Un dispositif à microprocesseur permet de déduire l'influence de cette filiation dans le canal de mesure du plutonium. La résolution des pics peut éventuellement être améliorée par une grille de collimation. (Balise Pu BFSA, DFF Pu 12 et 30, DADA, Eberline, Radeco,...).

Spectrométrie $\alpha$ sous vide partiel. Le principe est le même que celui ci-dessus mais le vide partiel régnant dans la chambre de mesure améliore la résolution. La séparation des pics entre les émetteurs artificiels et naturels est donc meilleure et facilite ainsi la déduction de l'influence des éléments naturels dans le canal de mesure du plutonium (APIA KIMMEL).

Vitesse de variation de la contamination. L'évolution de la contamination due aux éléments naturels est, en général, relativement lente. Un dispositif électronique permet donc une distinction entre cette évolution lente supposée être due à des éléments naturels et une évolution rapide qui peut être due à une contamination par du $\mathrm{Pu}$; ce type d'électronique peut s'adapter à différents modèles d'appareils (MAFF, DFF $\alpha$, DPA Cadarache, EAR $761, \ldots)$. II s'agit du signaleur de contamination rapide mis au point par la Section d'électronique et d'instrumentation (SEIn) Cadarache.

Impacteur et mesure $\alpha$ globale. La discrimination est faite grâce à un impacteur : les particules de $\mathrm{Pu}$ étant plus grosses que celles des élé- 
ments naturels viennent, seules, se piéger sur un film scintillant adhésif. II suffit alors de faire une mesure $\alpha$ globale (MD Pu).

Impacteur et spectrométrie $\alpha$. Le fait d'allier deux des principes précédents paraissait séduisant. L'impaction se faisait sur ruban adhésif de bureau. Le principe essayé il y a environ 15 ans par un constructeur anglais est resté sans suite, vraisemblablement pour des raisons technologiques.

Séparation dichotomique. La séparation s'effectue avec deux filtres fibreux de pouvoirs d'arrêt différents (filtres bleu et rose) et deux mesures associées. Elle peut également se faire sur une membrane de type Nuclépore avec un seul détecteur. La porosité est telle que le dépôt actif des descendants du radon et du thoron traverse le filtre ou la membrane alors que le plutonium est retenu. La mesure s'effectue alors en $\alpha$ global (double mandarin).

Electronique. Tous les appareils comportent maintenant un dispositif à microprocesseur permettant d'effectuer les opérations nécessaires de façon rapide et fiable, et en particulier la correction radon ou thoron. Les algorithmes utilisés au CEA sont ceux du SPR, Saclay (M. CASTRI) ou du SEIn, Cadarache (MM. CAROSSI et DA COSTA VEIRA).

Dispositif d'aspiration. II est possible d'utiliser soit : une pompe par appareil avec éventuellement un venturi qui assure un débit constant (BFSA), soit un pompage centralisé (centrale Caspa, par exemple) qui réduit le nombre des pompes et le bruit dans les locaux et permet éventuellement une reprise automatique de secours en cas de panne. II est, en revanche, nécessaire de mettre en place un réseau de tuyauteries.

Appareils de prélèvement. Citons, pour mémoire, les appareils de prélèvement permettant des contrôles a posteriori. Ces appareils peuvent être individuels (Casella, Siersaton, AC 5 Garnier) ou collectifs. A noter également les dispositifs permettant d'effectuer des granulométries en local (type cascade impactor, par exemple). Les mesures sur ces filtres peuvent être faites par la méthode des comptages successifs afin d'avoir un résultat valable sans attendre la décroissance complète des éléments naturels (SPR, Saclay: M. LEBLANC).

\section{TENDANCES ACTUELLES}

Une meilleure connaissance des conditions de ventilation des locaux est possible par simulation soit avec des gaz tels que l'hélium (DPT, Saclay) ou le SF 6 (Laboratoire d'études d'environnement et de sécurité industrielle, LESI, Grenoble) soit avec des aérosols d'uranine par exemple. Tous les SPR pourraient employer cette dernière méthode, après modification du générateur utilisé pour les tests in situ des ensembles filtrants à haute efficacité (le diamètre des aérosols d'essai devrait être de 2 à $3 \mu \mathrm{m}$ au lieu de $0,15 \mu \mathrm{m}$ sur l'appareil actuel).

Cette connaissance ajoutée à celle découlant des conditions d'exploitation permettra une meilleure détermination des points sensibles, donc des points de prélèvement. On risque malheureusement d'être acculé à un dilemme difficile à trancher : si les mouvements de l'air dans le local sont importants, l'homogénéité est bonne et un seul point de contrôle peut suffire (mais le local est entièrement contaminé) ; si les mouvements de l'air sont très faibles, la dispersion de la contamination est bien moindre, 
le local peut n'être que partiellement contaminé, mais il faut alors multiplier les points de contrôle pour qu'un incident ne puisse pas passer inaperçu.

Dans le cas où les conditions opératoires et de ventilation sont mal définies, la tendance va soit à la multiplication des points de prélèvement (avec contrôle a posteriori) soit à l'accroissement du nombre des points de contrôle (à condition de disposer d'un ensemble de détection à prix compétitif tel le DPA SPR, Cadarache, par exemple).

Pour ce qui concerne les chantiers, le besoin se fait sentir d'appareillage mobile de faible poids et encombrement pouvant être mis en place par un seul agent. Cet ensemble doit être doté d'une alarme éventuellement raccordable au tableau de contrôle des rayonnements (TCR).

En complément au système précédent, il est possible de doter les agents d'appareils de prélèvement individuels (contrôle a posteriori) et bientôt d'appareils d'alarme individuels [2]. A remarquer, toutefois, que ce type d'appareil amène une certaine gêne au porteur du fait de son poids, de son encombrement et du bruit ; s'il est parfois mal admis en tant que prélèvement, peut-être sera-t-il mieux toléré en tant qu'alarme. II serait surtout utile, dans ce dernier cas, aux agents des équipes d'intervention qui, dans un local, n'ont pas de poste de travail bien défini.

Le rapport de J. CHARUAU [1] montre, si besoin était, la nécessité d'optimiser les conduits de prélèvement quant à leur longueur, leur diamètre, leur rayon de courbure, leur nature et le fini intérieur des parois. II convient donc de faire systématiquement l'étude du réseau de canalisation, étant bien entendu qu'il est nécessaire de faire des hypothèses sur la granulométrie des aérosols qui seront générés en cas d'incident ou d'accident. Tous les essais permettant de faire progresser les connaissances sur la granulométrie des aérosols de plutonium en conditions accidentelles sont évidemment du plus haut intérêt. De tels essais ont été en particulier effectués à La Hague [3].

Dans le cas où les mouvements de l'air dans le local sont bien connus, et si le travail d'exploitation est uniquement un travail de routine parfaitement maîtrisé, il devrait être possible de se contenter d'un nombre restreint de points de prélèvement. Au besoin, un prélèvement en gaine d'extraction pourrait alors couvrir des cas d'incidents ou d'accidents particuliers. II est alors nécessaire de disposer d'un appareil aussi performant que possible. Pour cela, la tendance est à l'accroissement du débit de prélèvement $\left(0,8\right.$ à $1 \mathrm{~m}^{3} \cdot \mathrm{min}^{-1}$ sur la BFSAB) et à la discrimination des éléments naturels dus à la filiation radon-thoron. Pour cela, deux dispositifs principaux sont actuellement en concurrence : il s'agit de la spectrométrie $\alpha$ et de la pseudo coïncidence $\alpha$ - $\beta$. A noter, toutefois, un troisième système qui s'avère intéressant pour de petites installations: la tête DPA (SPR, Cadarache) avec un signaleur de contamination rapide.

La spectrométrie $\alpha$ est bien connue et largement utilisée sur de nombreux appareils. Les difficultés proviennent :

- de la nécessité d'avoir des membranes filtrantes de bonne qualité (pour une question de résolution) qui limitent le débit de prélèvement ;

- de la perte de résolution due à l'empoussiérement du filtre, ce qui amène un empiétement des rayonnements "parasites" des descendants radon et thoron dans la voie $\mathrm{Pu}$; 
- de la difficulté de soustraire les dits éléments parasites dans une proportion qui peut évoluer au cours du temps. Ce problème se résout actuellement grâce à des algorithmes mis au point pour les unités de traitement.

A noter que la résolution peut être améliorée par la mise en dépression de la cellule de mesure (avec, en contre partie, une limitation du débit) ou par l'utilisation d'une grille de collimation (diminution de rendement due à l'éloignement de la source).

La méthode de pseudo coïncidence $\alpha-\beta$ était déjà utilisée sur les EAR 900 et 901 . Le reproche fait à ces appareils était surtout une instabilité de l'électronique. Des améliorations importantes ont été apportées et la méthode utilisée sur des appareils allemands est également mise en $œ u v r e$ sur la balise à grande sensibilité BFSAB [4]. La difficulté résidait, pour ce procédé, dans la fixation du rapport $\beta / \alpha$ pour les éléments naturels.

La tête DPA (SPR, Cadarache) est utilisée dans certains établissements associée à une sonde MIP 10 avec un signaleur de contamination rapide et un réseau d'aspiration; elle peut constituer un système de détection à un prix abordable pour de petites installations. II doit être possible de perfectionner ce dispositif en utilisant une ou plusieurs sondes "extérieures" spécifiques pour le radon/thoron et un traitement d'information plus perfectionné. L'inconvénient majeur réside dans le changement manuel des filtres.

Fait important à noter, l'avènement des unités de traitement à microprocesseur autorise des performances nettement en progrès par rapport à l'électronique classique. Ceci est à l'origine de l'accroissement attendu de la sensibilité des nouvelles balises.

\section{CONCLUSION}

Ce qui précède montre l'éventail des possibilités qui sont offertes aux concepteurs d'installations de détection de contamination atmosphérique. En fait, il est à constater que la solution aux problèmes posés est fonction des données de base : configuration des lieux, nombre des points de contrôle, réseau de ventilation avec préfiltration ou non.

Ces différents paramètres vont permettre la détermination d'une solution où le rapport qualité - prix sera optimal s'ils sont pris en compte d'une façon judicieuse.

Ainsi, pour une installation comportant un nombre important de points à surveiller, la formule de la centrale d'aspiration avec prélèvement et comptage au niveau de chaque poste paraît particulièrement séduisante, car on s'affranchit ainsi du problème du transport des aérosols. Certes, des servitudes demeurent comme le remplacement des filtres de collecte. Toutefois, cet handicap semble facile à surmonter avec l'utilisation du système "chargeur" mis au point pour d'autres applications. La gêne occasionnée par l'influence de la radioactivité naturelle peut être facilement résolue, grâce à des mesures de référence introduites sans difficulté majeure dans un logiciel bien adapté. 
Néanmoins, il faut avoir bien présent à l'esprit que l'essentiel n'est pas d'effectuer des mesures à comparer aux inhalations possibles des opérateurs mais de rechercher un dispositif, aussi sensible et fiable que possible, capable de donner une alarme sur une situation anormale.

\section{RÉFÉRENCES}

[1] CHARUAU J. Etude du dépôt des particules dans les conduits. Optimisation des tubes de prélèvement des aérosols radioactifs (Thèse, Paris VI, 1981). Rapport CEA-R- 5158, 1982.

[2] CHARUAU J., PESCAYRE G., PRIGeNT R. Moniteur individuel de la contamination atmosphérique alpha (type MONICA $\alpha$ ). Journées d'études SFRP "Plutonium et radioprotection, Saclay, 14-16 juin 1983. Radioprotection, 1984, 19, 1-13.

[3] GOUGUET J. Corrélation existant entre les caractéristiques du plutonium issu d'une usine de retraitement et les diverses grandeurs utilisées en radioprotection, en fonction de divers paramètres. Journées d'études SFRP "Plutonium et radioprotection, Saclay, 14-16 juin 1983 (à paraître).

[4] MATUTANO J. Présentation d'une nouvelle balise de mesure de la contamination dans l'environnement, Journées d'études SFRP "Plutonium et radioprotection". Saclay, 14-16 juin 1983 (à paraître).

[5] SOCIETE FRANCAISE DE RADIOPROTECTION (SFRP). Journées d'études "Plutonium et radioprotection", Saclay, 14-16 juin 1983, Session 4: Balise plutonium (à paraître). 